\title{
Cancer Causation Research
}

National Cancer Institute

\section{Source}

National Cancer Institute. Cancer Causation Research. NCI Thesaurus. Code C15897.

Cancer causation research studies the events involved in the initiation and promotion of cancer. It encompasses chemical and physical carcinogenesis, biological carcinogenesis, epidemiology, chemoprevention, and nutrition research. Studies focus on external agents such as chemicals, radiation, fibers and other particles, viruses, parasitic infections, and host factors such as hormone levels, nutritional and immunologic status, and the genetic endowment of the individual, all of which contribute to the initiation and promotion of cancer. 